\title{
A Complete Fisher Discriminant Analysis for Based Image Matrix and Its Application to Face Biometrics
}

\author{
R.M. Mutelo, W.L. Woo, and S.S. Dlay \\ School of Electrical, Electronic and Computer Engineering \\ University of Newcastle \\ Newcastle upon Tyne, NE1 7RU \\ United Kingdom \\ \{risco.mutelo,w.1.woo,s.s.dlay\}@ncl.ac.uk
}

\begin{abstract}
This paper presents a Complete Orthogonal Image discriminant (COID) method and its application to biometric face recognition. The novelty of the COID method comes from 1) the derivation of two kinds of image discriminant features, image regular and image irregular, in the feature extraction stage and 2) the development of the Complete OID (COID) featuresbased on the fusion of the two kinds of image discriminant features used in classification. Firstly, the COID method first derives a feature image of the face image with reduced dimensionality of the image matrix by means of two dimensional principal component analysis and then performs discriminant analysis in a double discriminant subspaces in order to derive the image regular and irregular features making it more suitable for small sample size problem. Finally combines the image regular and irregular features which are complementary for achieving better discriminant features. The feasibility of the COID method has been successfully tested using the ORL images where it was $73.8 \%$ more superior to 2DFLD method on face recognition.
\end{abstract}

Keywords: Biometrics, Face Recognition, Fisher Discriminant Analysis (FDA), two dimensional Image, Feature extraction, image representation.

\section{Introduction}

Over the last few years, biometrics has attracted a high interest in the security technology marketplace. Biometric technologies use physiological or behavioural characteristics to identify and authenticate users attempting to gain access to computers, networks, and physical locations. The fastest growing areas of advanced security involve biometric face recognition technologies. Biometric face recognition technology offers great promise in its ability to identify a single face, from multiple lookout points, from a sea of hundreds of thousands of other faces. However building an automated face recognition system is very challenging. In real world applications, particularly in image recognition, there is a lot of small sample size (SSS) problems in observation space (input space). In such problems, the number of training samples is less than the dimension of feature vectors. A good face recognition methodology should consider representation as well as classification issues. Straightforward image 
projection techniques such two-dimensional principal component analysis (2DPCA) [1], two dimensional reduction PCA (2D RPCA) [2], two dimensional FLD [3, 4] are among the most popular methods for representation and recognition.

This paper introduces a novel Complete Orthogonal Image discriminant (COID) method for biometric face representation and recognition. The novelty of the COID method comes from 1) the derivation of two kinds of image discriminant features, image regular and image irregular, in the feature extraction stage and 2) the development of the Complete OID (COID) features based on the fusion of the two kinds of image discriminant features used in classification. In particular, the COID method first derives a feature image of the face image with reduced dimensionality of the image matrix by means of two dimensional principal component analysis and then performs discriminant analysis in a double discriminant subspaces in order to derive the image regular and irregular features making it more suitable for small sample size problem. The 2DPCA transformed face images preserves the spatial structure that defines the 2D face image and exhibit strong characteristics features with reduced noise and redundancies. Whereas, 2DFLD would further reduce redundancy and represent orthogonal discriminant features explicitly.

\section{Outline of 2DPCA and 2DFLD}

Given a set of $M$ training samples $\mathbf{X}_{1}, \mathbf{X}_{2}, \cdots, \mathbf{X}_{M}$ in the input space $\Re^{m \times n}$. The aim is to project image $\mathbf{X}_{i}$, an $m \times n$ face matrix, onto $\mathbf{B}_{i}$ by the following linear transformation

$$
\mathbf{Y}_{i}=\mathbf{X}_{i} \mathbf{B}
$$

Thus, we obtain an an $m \times d$ dimensional projected matrix $\mathbf{Y}_{i}$, which is the feature matrix of the image sample $\mathbf{X}_{i}$. In the 2DPCA method, the total scatter $\mathbf{S}_{t}$ of the projected samples is introduced to measure the discriminatory power of the projection vector $\mathbf{B}$. The total scatter of the projected samples can be characterized by the trace of the scatter matrix of the projected feature vectors. Whereas in 2DFLD the between class scatter $\mathbf{S}_{b}$ and the within-class scatter $\mathbf{S}_{w}$ matrices of the projected samples are introduced. From this point of view, we adopt the following criterion:

$$
\begin{gathered}
J\left(\beta_{i}\right)=\beta^{T} \mathbf{S}_{t} \beta \\
J\left(\varphi_{i}\right)=\frac{\varphi_{i}^{T} \mathbf{S}_{b} \varphi_{i}}{\varphi_{i}^{T} \mathbf{S}_{w} \varphi_{i}}, \varphi_{i} \neq 0
\end{gathered}
$$

where $\beta_{i}$ and $\varphi_{i}$ are the unitary vector that maximizes the 2DPCA criterion (2) and the 2DFLD criterion (3) which are called the optimal projection axis, $\beta_{o p t}$ and $\varphi_{o p t}$. In general, it is not enough to have only one optimal projection axis. We usually need to select a set of projection axes, $\mathbf{B}=\left\lfloor\beta_{1}, \beta_{2}, \cdots, \beta_{d}\right\rfloor$ and $\mathbf{B}=\left\lfloor\varphi_{1}, \varphi_{2}, \cdots, \varphi_{d}\right\rfloor$, subject to the orthonormal constraints. The scatter matrices can be defined as follows: 


$$
\begin{gathered}
\mathbf{S}_{t}=\frac{1}{M} \sum_{i=1}^{M}\left(\mathbf{X}_{i}-\mathbf{X}_{0}\right)^{T}\left(\mathbf{X}_{i}-\mathbf{X}_{0}\right) \\
\mathbf{S}_{b}=\frac{1}{M} \sum_{j=1}^{C} L_{i}\left(\overline{\mathbf{X}}_{i}-\mathbf{X}_{0}\right)^{T}\left(\overline{\mathbf{X}}_{i}-\mathbf{X}_{0}\right) \\
\mathbf{S}_{w}=\frac{1}{M} \sum_{i=1}^{C} \sum_{j=1}^{L_{l}}\left(\mathbf{X}_{i j}-\overline{\mathbf{X}}_{i}\right)^{T}\left(\mathbf{X}_{i j}-\overline{\mathbf{X}}_{i}\right)
\end{gathered}
$$

where $\mathbf{X}_{i j}$ denotes the $j$ th training sample in class $i, L_{i}$ is the number of training samples in class $i, \overline{\mathbf{X}}_{i}$ is the mean of the training samples in class $i, C$ is the number of image classes, $\mathbf{X}_{0}$ is the global mean across all training samples.

\section{Complete Orthogonal Image Discriminant Approach}

In this section, we examine the problem in the whole input space rather than in the space transformed by 2DPCA first.

\subsection{Fundamentals}

Suppose we have a set of $M$ training samples $\mathbf{X}_{1}, \mathbf{X}_{2}, \cdots, \mathbf{X}_{M}$ in the input space of size $m \times n$, by definition the $\operatorname{rank}\left(\mathbf{X}_{i}\right)=\min (m, n)$. From the denominator in (3), we have

$$
\begin{aligned}
\operatorname{rank}\left(\mathbf{S}_{w}\right) & =\operatorname{rank}\left(\frac{1}{M} \sum_{i=1}^{C} \sum_{j=1}^{L_{i}}\left(\mathbf{X}_{i j}-\overline{\mathbf{X}}_{i}\right)^{T}\left(\mathbf{X}_{i j}-\overline{\mathbf{X}}_{i}\right)\right) \\
& \leq(M-C) \cdot \min (m, n)
\end{aligned}
$$

So that $\mathbf{S}_{w}$ is non-singular when

$$
M \geq c+\frac{n}{\min (m, n)}
$$

If the within-class covariance operator $\mathbf{S}_{w}$ is invertible, $\varphi_{i}^{T} \mathbf{S}_{w} \varphi_{i}>0$ always holds for every nonzero vector $\varphi_{i}$. Therefore, the Fisher criterion can be directly employed to extract a set of optimal discriminant vectors. However, in some case it is almost impossible to make $\mathbf{S}_{w}$ invertible because of the limited amount of training samples in real-world applications. This means that there always exist vectors satisfying $\varphi_{i}^{T} \mathbf{S}_{w} \varphi_{i}=0$. In [5], it is shown that these vectors are from the null space of $\mathbf{S}_{w}$ for the vectorized fisher discriminant approach. These vectors turn out to be very effective if they satisfy $\varphi_{i}^{T} \mathbf{S}_{b} \varphi_{i}>0$ at the same time [5]. The positive image between-class scatter matrix makes the data become well separable when the within-class scatter matrix is 
zero. In such a case, the Fisher criterion degenerates into the following between-class covariance criterion:

$$
J_{b}\left(\varphi_{i}\right)=\varphi_{i}^{T} \mathbf{S}_{b} \varphi_{i},\left(\left\|\varphi_{i}\right\|=1\right)
$$

As a special case of the Fisher criterion, it is reasonable to use the image between class scatter to measure the discriminatory ability of a projection axis when the image within-class covariance is zero.

\subsection{Optimal Orthogonal Image Discriminant Vectors}

The input space $\Re^{m \times n}$ is relatively large and mostly empty (containing noise and redundancies), it is computationally intensive to calculate the optimal discriminant vectors directly. Our strategy is to reduce the feasible solution space (search space) where two kinds of discriminant vectors might hide. Suppose $\beta_{1}, \beta_{2}, \cdots, \beta_{d}$ are eigenvectors corresponding to the largest positive eigenvalues of $\mathbf{S}_{t}$. We can define the subspace $\boldsymbol{\psi}_{t}=\operatorname{span}\left\{\beta_{1}, \beta_{2}, \cdots, \beta_{d}\right\}$ and its orthogonal complementary space is denoted by $\boldsymbol{\psi}_{\frac{1}{t}}=\operatorname{span}\left\{\beta_{d+1}, \beta_{d+2}, \cdots, \beta_{n}\right\}$. The eigenvectors $\left\{\beta_{d+1}, \beta_{d+2}, \cdots, \beta_{n}\right\}$ are relatively close to zero. In 2DPCA these eigenvectors are considered to contain very little or no discriminant information. Since $n=\operatorname{rank}\left(\mathbf{S}_{t}\right)$, non of the eigenvalues of $\mathbf{S}_{t}$ are negative. Therefore, we refer to $\boldsymbol{\psi}_{\frac{1}{t}}$ in this paper as the positive null space of $\mathbf{S}_{t}$. Since $\mathfrak{R}^{m \times n}=\boldsymbol{\psi}_{t} \oplus \boldsymbol{\psi}_{\frac{1}{t}}$, it follows that for an arbitrary vector $\varphi_{i} \in \mathfrak{R}^{m \times n}, \varphi_{i}$ can be uniquely represented in the form $\varphi_{i}=\phi_{i}+\varsigma_{i}$ with $\boldsymbol{\varphi}_{i} \in \boldsymbol{\psi}_{t}$ and $\varsigma_{i} \in \boldsymbol{\psi}_{\frac{1}{t}}$. We can define the mapping.$\Re^{m \times n} \rightarrow \boldsymbol{\psi}_{t}$ using

$$
\varphi_{i}=\phi_{i}+\varsigma_{i} \rightarrow \phi_{i}
$$

where $\phi_{i}$ is the orthogonal projection of $\varphi_{i}$ onto $\psi_{t}$. The eigenvectors $\varsigma_{i}$ have eigenvalues relatively close to zero, thus discarded in 2DPCA. It follows that $J\left(\varphi_{i}\right)=J\left(\phi_{i}\right)$. Since the new search space is much smaller (less dimensionality), it is easer to derive discriminant vectors from it. The aim at this point is to calculate Fisher optimal discriminant vectors in the reduced search space $\boldsymbol{\psi}_{t}$. According to linear algebra theory [6], $\boldsymbol{\psi}_{t}$ is isomorphic to $m \times d$ dimensional matrix space $\mathfrak{R}^{m \times d}$. The corresponding isomorphic mapping is

$$
\varphi_{i}=\mathbf{B} \eta_{i}, \text { where } \mathbf{B}=\left(\beta_{1}, \beta_{2}, \cdots, \beta_{d}\right), \eta_{i} \in \mathfrak{R}^{m \times d}
$$

Under the isomorphic mapping $\varphi_{i}=\mathbf{B} \eta_{i}$, the criterion function (3) and (9) in the subspace is, converted into

$$
J\left(\varphi_{i}\right)=\frac{\eta_{i}^{T}\left(\mathbf{B}^{T} \mathbf{S}_{b} \mathbf{B}\right) \eta_{i}}{\eta_{i}^{T}\left(\mathbf{B}^{T} \mathbf{S}_{w} \mathbf{B}\right) \eta_{i}} \text { and } J_{b}\left(\varphi_{i}\right)=\eta_{i}^{T}\left(\mathbf{B}^{T} \mathbf{S}_{b} \mathbf{B}\right) \eta_{i}
$$




$$
J\left(\eta_{i}\right)=\frac{\eta_{i}^{T} \hat{\mathbf{S}}_{b} \eta_{i}}{\eta_{i}^{T} \hat{\mathbf{S}}_{w} \eta_{i}}, \eta_{i} \neq 0 \text { and } J_{b}\left(\eta_{i}\right)=\eta_{i}^{T} \hat{\mathbf{S}}_{b} \eta_{i},\left\|\eta_{i}\right\|=1
$$

where $\hat{\mathbf{S}}_{b}=\mathbf{B}^{T} \mathbf{S}_{b} \mathbf{B}$ and $\hat{\mathbf{S}}_{w}=\mathbf{B}^{T} \mathbf{S}_{w} \mathbf{B}$. This means that $J\left(\eta_{i}\right)$ is a generalized Rayleigh quotient and $J_{b}\left(\eta_{i}\right)$ Rayleigh quotient in the isomorphic space $\Re^{m \times d}$. Now, the problem of calculating the optimal discriminant vectors in subspace $\boldsymbol{\psi}_{t}$ is transformed into the extremum problem of the (generalized) Rayleigh quotient in the isomorphic space $\Re^{m \times d}$. Therefore we can obtain the discriminant feature matrix $\boldsymbol{\Pi}_{j}$ by the following transformation:

$$
\boldsymbol{\Pi}_{j}=\mathbf{X}_{j} \mathbf{T}
$$

$$
\begin{aligned}
& \text { where } \mathbf{T}=\left(\varphi_{1}, \varphi_{2}, \cdots, \varphi_{q}\right)=\left(\mathbf{B} \eta_{1}, \mathbf{B} \eta_{2}, \cdots, \mathbf{B} \eta_{q}\right) \\
& =\mathbf{B}\left(\eta_{1}, \eta_{2}, \cdots, \eta_{q}\right)
\end{aligned}
$$

The transformation in (14) can be divided into two transformations:

$$
\begin{gathered}
\mathbf{Y}_{j}=\mathbf{X}_{j} \mathbf{B}, \text { where } \mathbf{B}=\left(\beta_{1}, \beta_{2}, \cdots, \beta_{d}\right) \\
\boldsymbol{\Pi}_{j}=\mathbf{Y}_{j} \boldsymbol{\Lambda}, \text { where } \boldsymbol{\Lambda}=\left(\eta_{1}, \eta_{2}, \cdots, \eta_{q}\right)
\end{gathered}
$$

The transformation (15) is exactly 2DPCA [1]. Looking back at (13) and considering the two matrices $\hat{\mathbf{S}}_{b}$ and $\hat{\mathbf{S}}_{w}$ are the between class and within-class scatter matrices in $\Re^{m \times d}$. Therefore, 2DPCA is firstly used to reduce dimension of the input space. Low dimensionality is important for learning, performing 2DFLD on the transformed images results in better generalization to unseen images.

\subsection{Two Kinds of Image Discriminant Features}

Our strategy is to split the space $\Re^{m \times d}$ into two subspaces: the positive null space and the range space of $\hat{\mathbf{S}}_{w}$ and then use the Fisher criterion to derive the image regular discriminant features from the range space and use the between-class scatter criterion to derive the image irregular discriminant matrices from the null space. Suppose $\alpha_{1}, \alpha_{2}, \cdots, \alpha_{d}$ are the orthonormal eigenvectors of $\hat{\mathbf{S}}_{w}$ and the first $q$ ones correspond to largest positive eigenvalues. We define the subspace, $\boldsymbol{\Theta}_{w}=\operatorname{span}\left\{\alpha_{1}, \alpha_{2}, \cdots, \alpha_{q}\right\}$, the ranger space and it's orthogonal complementary space is $\boldsymbol{\Theta}_{\frac{1}{w}}=\operatorname{span}\left\{\alpha_{q+1}, \alpha_{d+2}, \cdots, \alpha_{d}\right\}$. Since for the nonzero vector $\eta_{i}$ in $\boldsymbol{\Theta}_{\frac{1}{w}}$, the image within class scatter and between class scatter becomes $\eta_{i}^{T} \hat{\mathbf{S}}_{w} \eta_{i}=0$ and $\eta_{i}^{T} \hat{\mathbf{S}}_{b} \eta_{i}>0$. Therefore the Fisher criterion $J_{b}\left(\eta_{i}\right)=\eta_{i}^{T} \hat{\mathbf{S}}_{b} \eta_{i}$ is used to derive the discriminant feature matrix. On the other hand, for every nonzero vector $\eta_{i}$ in $\boldsymbol{\Theta}_{w}$ satisfies $\eta_{i}^{T} \hat{\mathbf{S}}_{w} \eta_{i}>0$, it is feasible to derive the optimal regular discriminant vectors from $\boldsymbol{\Theta}_{w}$ using the standard Fisher criterion $J\left(\eta_{i}\right)$. To calculate the optimal image regular discriminant vectors in $\boldsymbol{\Theta}_{w}$. The 
dimension of $\boldsymbol{\Theta}_{w}$ is $m \times q$, space $\boldsymbol{\Theta}_{w}$ is isomorphic to the matrix space $\Re^{m \times q}$ and the corresponding isomorphic mapping is

$$
\eta=\mathbf{B}_{1} \xi, \text { where } \mathbf{B}_{1}=\left(\alpha_{1}, \alpha_{2}, \cdots, \alpha_{q}\right)
$$

Under this mapping, the Fisher criterion $J\left(\eta_{i}\right)$ is converted into

$$
\bar{J}\left(\xi_{i}\right)=\frac{\xi_{i}^{T} \tilde{\mathbf{S}}_{b} \xi_{i}}{\xi_{i}^{T} \tilde{\mathbf{S}}_{w} \xi_{i}}, \xi_{i} \neq 0
$$

where $\widetilde{\mathbf{S}}_{b}=\mathbf{B}_{1}^{T} \hat{\mathbf{S}}_{b} \mathbf{B}_{1}$ and $\widetilde{\mathbf{S}}_{w}=\mathbf{B}_{1}^{T} \hat{\mathbf{S}}_{w} \mathbf{B}_{1}$. Thus the generalized eigenvector of the generalized eigenequation is $\tilde{\mathbf{S}}_{b} \boldsymbol{\xi}_{i}=\lambda_{i} \tilde{\mathbf{S}}_{w} \boldsymbol{\xi}_{i}$. Let $\vartheta_{1}, \vartheta_{2}, \cdots, \vartheta_{f}$ be the $f$ largest eigenvectors of $\tilde{\mathbf{S}}_{w}^{-1} \widetilde{\mathbf{S}}_{b}$, we can obtain the image regular discrminant vectors $\bar{\eta}_{i}=\mathbf{B}_{1} \vartheta_{i}, i=1, \cdots, f$ using (17). Similarly, the optimal image irregular discriminant vectors within $\boldsymbol{\Theta}_{\frac{1}{w}}$ is isomorphic to the matrix space $\mathfrak{R}^{m \times(d-q)}$ and the corresponding isomorphic mapping is

$$
\eta=\mathbf{B}_{2} \xi, \text { where } \mathbf{B}_{2}=\left(\alpha_{q+1}, \alpha_{q+2}, \cdots, \alpha_{d}\right)
$$

Under this mapping, the Fisher criterion $\hat{J}_{b}\left(\eta_{i}\right)$ is converted into

$$
\hat{J}_{b}\left(\xi_{i}\right)=\xi_{i} \overrightarrow{\mathbf{S}}_{b} \xi_{i},\left\|\xi_{i}\right\|=1
$$

where $\overrightarrow{\mathbf{S}}_{b}=\mathbf{B}_{2}^{T} \hat{\mathbf{S}}_{b} \mathbf{B}_{2}$. Letting $v_{1}, v_{2}, \cdots, v_{f}$ be the eigenvectors of $\overrightarrow{\mathbf{S}}_{b}$ corresponding to $f$ largest eigenvalues. We have $\hat{\eta}_{i}=\mathbf{B}_{2} v_{i}$ the optimal irregular discriminant vectors with respect to $\hat{J}_{b}(\eta)$. The linear discriminant transformation in (16) can be performed in $\Re^{m \times d}$. After the projection of the image sample $\mathbf{Y}_{j}$ onto the regular discriminant vectors $\bar{\eta}=\left(\bar{\eta}_{1}, \bar{\eta}_{2}, \cdots, \bar{\eta}_{f}\right)$, we can obtain the image regular discriminant feature matrix:

$$
\mathbf{\Pi}^{1}=\mathbf{Y}_{j}\left(\bar{\eta}_{1}, \bar{\eta}_{2}, \cdots, \bar{\eta}_{f}\right)=\mathbf{X}_{j} \mathbf{B}_{1} \vartheta
$$

where $\mathbf{B}_{1}=\left(\alpha_{1}, \alpha_{2}, \cdots, \alpha_{q}\right), \vartheta=\left(\vartheta_{1}, \vartheta_{2}, \cdots, \vartheta_{f}\right)$. After the projection of the sample $\mathbf{Y}_{j}$ onto the irregular discriminant vectors $\hat{\eta}_{1}, \hat{\eta}_{2}, \cdots, \hat{\eta}_{f}$, we can obtain the image irregular discriminant feature matrix:

$$
\boldsymbol{\Pi}^{2}=\mathbf{Y}_{j}\left(\hat{\eta}_{1}, \hat{\eta}_{2}, \cdots, \hat{\eta}_{f}\right)=\mathbf{X}_{j} \mathbf{B}_{2} \mathbf{v}
$$

where $\mathbf{B}_{2}=\left(\alpha_{q+1}, \alpha_{q+2}, \cdots, \alpha_{d}\right), v=\left(v_{1}, v_{2}, \cdots, v_{f}\right)$.

\subsection{Fusion of Two Kinds of Discriminant Features}

We propose a simple fusion strategy based on a summed normalized distance. The distance between two arbitrary feature matrices, 


$$
D\left(\boldsymbol{\Pi}^{i}, \boldsymbol{\Pi}^{j}\right)=\sum_{z=1}^{f} \sqrt{\left(\sum_{k=1}^{q}\left(\mathbf{T}_{z k}^{i}-\mathbf{T}_{z k}^{j}\right)^{2}\right)}
$$

where $\boldsymbol{\Pi}^{i}=\left[\mathbf{T}_{1}^{i}, \mathbf{T}_{2}^{i}, \ldots . \mathbf{T}_{f}^{i}\right]$ and $\boldsymbol{\Pi}^{j}=\left[\mathbf{T}_{1}^{j}, \mathbf{T}_{2}^{j}, \ldots . \mathbf{T}_{f}^{j}\right]$. For a given image sample we denote a feature matrix $\Pi=\left[\Pi^{1}, \Pi^{2}\right]$, where $\Pi^{1}, \quad \Pi^{2}$ are the regular and irregular discriminant feature matrices of the same pattern. The summed normalized-distance between sample $\boldsymbol{\Pi}$ and the training sample $\boldsymbol{\Pi}_{r}=\left[\boldsymbol{\Pi}_{r}^{1}, \boldsymbol{\Pi}_{r}^{2}\right]$ is defined by

$$
\bar{D}\left(\boldsymbol{\Pi}, \boldsymbol{\Pi}_{r}\right)=\theta_{r} \frac{D\left(\boldsymbol{\Pi}^{1}, \boldsymbol{\Pi}_{r}^{1}\right)}{\sum_{g=1}^{M} D\left(\boldsymbol{\Pi}^{1}, \boldsymbol{\Pi}_{g}^{1}\right)}+\theta_{i} \frac{D\left(\boldsymbol{\Pi}^{2}, \boldsymbol{\Pi}_{r}^{2}\right)}{\sum_{g=1}^{M} D\left(\boldsymbol{\Pi}^{2}, \boldsymbol{\Pi}_{g}^{2}\right)}
$$

where $\theta_{r}$ and $\theta_{i}$ are the fusion coefficient. These coefficients determine the weight of regular discriminant information and irregular information in the decision level.

\section{Results and Discussions}

Our analysis was performed on the Olivetti Research Laboratory (ORL) face database which contains 40 persons, each person has 10 different images. For some subjects, the images were taken at different times, which contain quite a high degree of variability in lighting, facial expression (open/closed eyes, smiling/nonsmiling etc), pose (upright, frontal position etc), scale, and facial details (glasses/no glasses). Examples of sample images from the ORL face database are shown in Fig. 1.

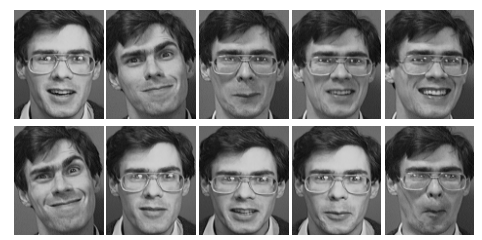

Fig. 1. Example ORL images with spatial resolution. $112 \times 92$ Note that the images vary in pose, size, and facial expression.

\subsection{ORL Face Database}

Firstly, an analysis was performed using one image samples per class for training. Thus, the total number of training samples is 40 and testing samples is 360 . That is, the total number of training samples and number of image classes are both 40 . According to (8) the image within scatter matrix $\hat{\mathbf{S}}_{w}$ is singular. It is almost impossible to make $\tilde{\mathbf{S}}_{w}$ invertible because of the limited amount of training samples used here as it is the usual case in real-world applications. That is, there always exist vectors satisfying $\xi_{i} \widetilde{\mathbf{S}}_{w} \xi_{i}=0$. The 2DLDA method is inapplicable since $\widetilde{\mathbf{S}}_{w}$ is 


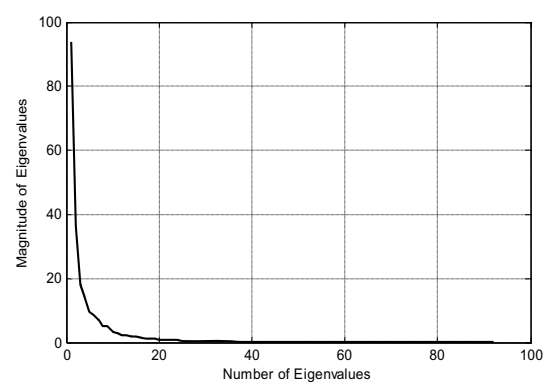

Fig. 2. Mmagnitudes of the eigenvalues in descending order from $\mathbf{S}_{t}$ for $2 \mathrm{DPCA}$

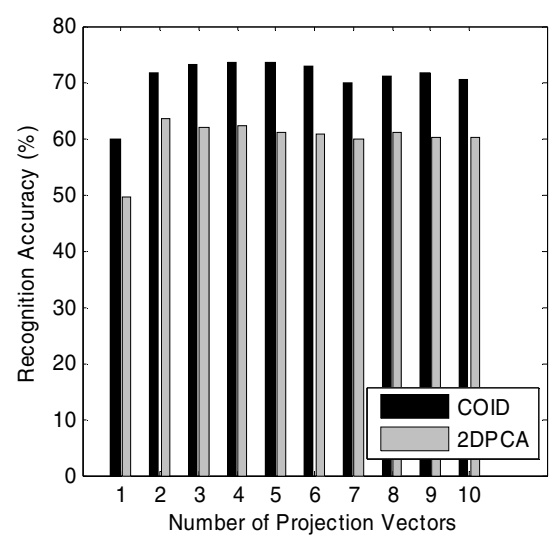

Fig. 3. Face Recognition performance for the COID method and 2DPCA. The 2DFLD method is inapplicable when a limited number of training samples is given.

singular thus suffers from the SSS problem. Therefore 2DPCA and COID methods were used for feature extraction. The size of image scatter matrix $\mathbf{S}_{t}$ was $92 \times 92$ and the $\operatorname{rank}\left(\mathbf{S}_{t}\right)=92$, the largest $d$ principal components are used as projection axes. According to section 3.2, most of the discriminant information is reserved. From Fig. 2, it is reasonable to use the 10 eigenvectors corresponding to the largest eigenvalues. The 2DPCA transformed feature matrix $\mathbf{Y}_{j}$ is of size $112 \times 10$, thus, it follows that $\widetilde{\mathbf{S}}_{b}$ and $\widetilde{\mathbf{S}}_{w}$ are $10 \times 10$. However, the $\operatorname{rank}\left(\hat{\mathbf{S}}_{w}\right)=0$, therefore the positive null space $\boldsymbol{\Theta}_{\frac{1}{w}}$ is used to extract the optimal discriminant vector where $\mathbf{B}_{1}=0$ and $\mathbf{B}_{2}=\left(\boldsymbol{\alpha}_{1}, \boldsymbol{\alpha}_{2}, \cdots, \boldsymbol{\alpha}_{10}\right)$ since $q=0$. The results in Fig. 3 lead to the following findings: 1) the classification performance with the COID outputs is better than that achieved by the 2DPCA method. The best performance of $73.6 \%$ was achieved by COID and 2DPCA obtained an accuracy of 63.6\%. The difference is that COID evaluates a smaller discriminant scatter matrix $\overrightarrow{\mathbf{S}}_{b}$ of size $10 \times 10$ more accurately, in 
Table 1. CPU Times (seconds) for feature extraction at top recognition accuracy for training

\begin{tabular}{|c|l|l|c|c|c|}
\hline & 2DPCA & 2DLDA & $\begin{array}{c}\text { Image } \\
\text { Irregular }\end{array}$ & $\begin{array}{c}\text { Image } \\
\text { Regular }\end{array}$ & COID \\
\hline Accuracy (\%) & 86.9 & 88.0 & 77.5 & 84.38 & 89.9 \\
\hline Dimensions & $112 \times 4$ & $112 \times 2$ & $112 \times 2$ & $112 \times 2$ & $112 \times 2$ \\
\hline $\begin{array}{c}\text { Feature Extraction } \\
\text { time (seconds) Pentium } \\
I \mathrm{~V} \quad \text { 3.00GHz, RAM } \\
496 \mathrm{Mb}\end{array}$ & 0.438 & 0.562 & 1.143 & 1.260 & 2.150 \\
\hline
\end{tabular}

contrast to the scatter matrix of 2DPCA of size $92 \times 92$. 2) The recognition accuracy increases as the number of eigenvectors $\mathbf{B}_{2}=\left(\boldsymbol{\alpha}_{1}, \boldsymbol{\alpha}_{2}, \cdots, \boldsymbol{\alpha}_{10}\right)$ used are increased. In particular, the face recognition perform of COID becomes stable when 4 and 5 eigenvectors are used where a top recognition accuracy of $73.6 \%$. After which the recognition accuracy decreases as noise and redundancies are introduced by the smaller eigenvectors making the system unstable. Similar trend is also observed for 2DPCA.

We then analysed the performance of 2DPCA [1], 2DFLD [3] and COID when two image samples per class are used for training and all the remaining image samples per class for testing. Again 10 eigenvectors were used to transform the 2DPCA space. Then spaces $\boldsymbol{\Theta}_{w}=\operatorname{span}\left\{\alpha_{1}, \alpha_{2}, \cdots, \alpha_{4}\right\}$ and $\boldsymbol{\Theta}_{\frac{1}{w}}=\operatorname{span}\left\{\alpha_{5}, \alpha_{6}, \cdots, \alpha_{10}\right\}$ were defined as in section 3.2. The fusion coefficients $\theta_{r}=2$ and $\theta_{i}=0.8$ were used combine the two kinds of discriminant information. The results in table 1 lead to the findings: 1) the COID method performs better than 2DPCA, 2DLDA methods with an accuracy of $89.9 \%$ correct recognition when $112 \times 2$ features are used. In addition, the dimensions of the feature matrix for COID method are comparable to 2DPCA and 2DLDA. 2) Although the image regular features show superior performance to the irregular feature, they are complementary to each other as shown by the COID results. As the discriminatory power depends on both the within class and between class covariance matrices, regular feature contain more discriminatory information. However, COID (compared to 2DPCA and 2DLDA) takes more time for feature extraction as it requires a two phase process which derives the discriminant information from double discriminant subspace making it a more powerful discriminant approach.

\section{Conclusions}

A novel Complete Orthogonal Image discriminant (COID) is developed. The COID method performs discriminant analysis in double discriminant subspaces: regular and irregular. The novelty of the OID method comes from 1) the derivation of the two kinds of image discriminant features, image regular and image irregular, in the feature extraction stage and 2) the development of the Complete OID (COID) features-based on the fusion of the two kinds of image discriminant features used in classification. In particular, the COID method first derives a feature image of the face image with reduced dimensionality of the image matrix by means of two dimensional principal component analysis. This low dimensionality is important for the discriminant 
learning leading to better generalization to unseen images, as the number of examples required for attaining a given level of performance grows exponentially with the dimensionality of the underlying representation space. The reason behind integrating the 2DPCA and the 2DFLD representations are twofold. Firstly, the 2DPCA transformed face images preserves the spatial structure that defines the $2 \mathrm{D}$ face image and exhibit strong characteristics features with reduced noise and redundancies. Lastly, 2DFLD would further reduce redundancy and represent orthogonal discriminant features explicitly. Since the image regular and irregular features are complementary for achieving better discriminant features there combined together in order to enhance the performance. In addition, the results show that COID is more suitable for Small Sample Size (SSS) problem due to its double discriminant subspace.

\section{References}

[1] Yang, J., Zhang, D., Frangi, A.F., Yang, J.-y.: dimensional PCA: a new approach to appearance-based face representation and recognition. Pattern Analysis and Machine Intelligence, IEEE Transactions 26, 131-137 (2004)

[2] Mutelo, R.M., Khor, L.C., Woo, W.L., Dlay, S.S.: Two-dimensional reduction PCA: a novel approach for feature extraction, representation, and recognition, presented at Visualization and Data Analysis 2006 (2006)

[3] Li, M., Yuan, B.: 2D-LDA: A statistical linear discriminant analysis for image matrix. Pattern Recognition Letters 26, 527-532 (2005)

[4] Yang, J., Zhang, D., Yong, X., Yang, J.-y.: Two-dimensional discriminant transform for face recognition. Pattern Recognition 38, 1125-1129 (2005)

[5] Chen, L.-F., Liao, H.-Y.M., Ko, M.-T., Lin, J.-C., Yu, G.-J.: A new LDA-based face recognition system which can solve the small sample size problem. Pattern Recognition 33, 1713-1726 (2000)

[6] Kreyszig, E.: Introductory Functional Analysis with Applications. John Wiley \& Sons, Chichester (1978) 